\title{
Peptide-enhanced mRNA transfection in
}

\section{cultured mouse cardiac fibroblasts and direct reprogramming towards cardiomyocyte-like cells}

This article was published in the following Dove Press journal:

International Journal of Nanomedicine

6 March 2015

Number of times this article has been viewed

\author{
Kunwoo Lee ${ }^{1,2}$ \\ Pengzhi Yu ${ }^{3}$ \\ Nithya Lingampalli' \\ Hyun Jin Kim' \\ Richard Tang' \\ Niren Murthy ${ }^{1,2}$ \\ 'Department of Bioengineering, \\ University of California, Berkeley, \\ CA, USA; ${ }^{2}$ UC Berkeley and \\ UCSF Joint Graduate Program \\ in Bioengineering, Berkeley/San \\ Francisco, CA, USA; ${ }^{3} \mathrm{Gladstone}$ \\ Institute of Cardiovascular Disease, \\ San Francisco, CA, USA
}

Correspondence: Niren Murthy Department of Bioengineering, University of California, 284 Hearst Memorial Mining Building, Berkeley, CA, USA Tel +l 5106644577

Fax +I 5106425835

Email nmurthy@berkeley.edu
Abstract: The treatment of myocardial infarction is a major challenge in medicine due to the inability of heart tissue to regenerate. Direct reprogramming of endogenous cardiac fibroblasts into functional cardiomyocytes via the delivery of transcription factor mRNAs has the potential to regenerate cardiac tissue and to treat heart failure. Even though mRNA delivery to cardiac fibroblasts has the therapeutic potential, mRNA transfection in cardiac fibroblasts has been challenging. Herein, we develop an efficient mRNA transfection in cultured mouse cardiac fibroblasts via a polyarginine-fused heart-targeting peptide and lipofectamine complex, termed C-Lipo and demonstrate the partial direct reprogramming of cardiac fibroblasts towards cardiomyocyte cells. C-Lipo enabled the mRNA-induced direct cardiac reprogramming due to its efficient transfection with low toxicity, which allowed for multiple transfections of Gata4, Mef2c, and Tbx5 (GMT) mRNAs for a period of 2 weeks. The induced cardiomyocyte-like cells had $\alpha-M H C$ promoter-driven GFP expression and striated cardiac muscle structure from $\alpha$-actinin immunohistochemistry. GMT mRNA transfection of cultured mouse cardiac fibroblasts via C-Lipo significantly increased expression of the cardiomyocyte marker genes, Actc1, Actn2, Gja1, Hand2, and Tnnt2, after 2 weeks of transfection. Moreover, this study provides the first direct evidence that the stoichiometry of the GMT reprogramming factors influence the expression of cardiomyocyte marker genes. Our results demonstrate that mRNA delivery is a potential approach for cardiomyocyte generation.

Keywords: direct cardiac reprogramming, cardiac fibroblast transfection, mRNA transfection, cardiac regeneration

\section{Introduction}

Myocardial infarction is a major cause of death in the world. An attractive therapy for myocardial infarction is to reprogram endogenous fibroblasts into cardiomyocytes in vivo and induce de novo cardiac regeneration. ${ }^{1,2}$ Significant progress has been made towards understanding the factors needed for direct reprogramming of cardiac cells. Ieda et al demonstrated that the overexpression of three cardiac transcription factors, Gata4, Mef2c, and Tbx5 (GMT), in mouse cardiac and tail-tip fibroblasts was sufficient to generate cardiomyocyte-like cells. ${ }^{2}$ In addition, retroviral transfection of cardiac fibroblasts in vivo with GMT induced direct reprogramming of those cells into functional cardiomyocytes, and also improved cardiac function in coronary artery-ligated mice. ${ }^{1,3}$ However, despite these promising studies, the translation of direct reprogramming by transcription factors has been hampered by safety concerns regarding the usage of retroviruses and lentiviruses, which have multiple disadvantages including immunogenicity, insertional mutagenesis, and tumorigenicity. ${ }^{4-7}$ 
The development of nonviral methods to perform direct reprogramming is a key step in enabling the clinical applications of this therapeutic approach; hence, there is a great interest in achieving this. Nonviral methods to reprogram fibroblasts into pluripotent stem cells have been developed using plasmids, ${ }^{8}$ mRNAs, ${ }^{4,9,10}$ and proteins. ${ }^{5,11}$ In addition, the recent success of direct neuronal reprogramming using plasmids demonstrates the potential of nonviral methods for direct reprogramming. ${ }^{6}$ Although nonviral methods have shown great promise for direct reprogramming, a general and versatile nonviral method for inducing direct reprogramming still needs to be developed.

mRNA delivery has great potential for direct reprogramming and has several advantages over plasmid-based gene delivery. ${ }^{4,10}$ First, mRNA does not contain viral or bacterial promoter sequences that can induce innate immune responses. ${ }^{12}$ Therefore, multiple mRNA transfections can be performed on mammalian cells. Second, mRNA transfection is more effective than DNA transfection on primary cells because it is not dependent on nuclear delivery. ${ }^{13}$ Third, mRNA transfection can be used to identify the optimal ratio of transcription factors needed to generate mature and functioning cardiomyocytes. mRNA delivery allows for a fine control of transcription factor expression levels and a stoichiometric analysis explaining the effects of transcription factor levels on reprogramming, both insights that are not possible with viral vectors or plasmids. ${ }^{3,14}$
Despite the great potential of mRNA transfection for direct reprogramming, current methods for delivering mRNA lack the efficiency or biocompatibility that are necessary for direct reprogramming applications. Traditional mRNA transfection methods have low efficiency and high toxicity for primary cardiac cells. ${ }^{15,16}$ In addition, the toxicity of transfection reagents is a critical issue for cellular reprogramming because reprogramming requires multiple transfections over a period of weeks. ${ }^{4,17}$

In this report, we present the demonstration of mRNAinduced cardiomyocyte-like gene expression in cardiac fibroblasts that were transfected with cardiac reprogramming factors in a new mRNA transfection system, termed C-Lipo, composed of a heart-targeting peptide (CRPPR-R9) and lipofectamine. CRPPR-R9 includes three parts: a CRPPR heart-targeting sequence, a four-glycine linker sequence, and a nine-arginine sequence (Figure 1). CRPPR is a hearttargeting peptide sequence discovered by phage display, which has been shown to localize in the heart tissue of mice after intravenous injection. ${ }^{18,19}$ CRPPR-R9 is designed to electrostatically interact with negatively charged mRNA due to its nine positively charged arginine residues, and then trigger endocytosis of the complexed mRNA in cardiac fibroblasts. We show here that cardiac fibroblasts can be partially reprogrammed towards cardiomyocyte-like cells via a daily transfection of GMT mRNAs using the C-Lipo system.
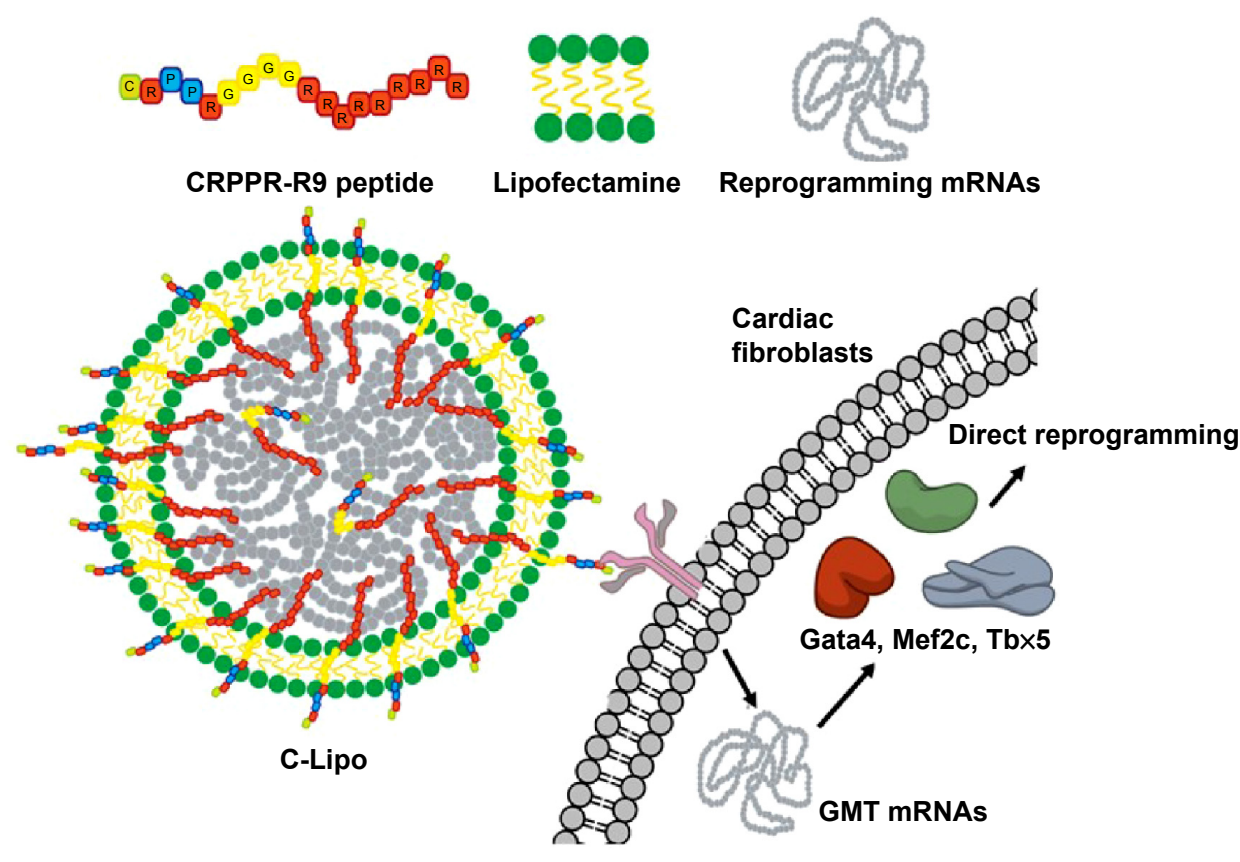

Figure I CRPPR-R9/lipofectamine/mRNA complex can deliver GMT mRNAs to cardiac fibroblasts and induct direct reprogramming towards cardiomyocyte-like cells. Notes: C-Lipo composed of a heart-targeting peptide (CRPPR-R9) and lipofectamine facilitates mRNA delivery in cardiac fibroblasts. GMT mRNA transfection induces partial direct reprogramming of cardiac fibroblasts towards cardiomyocytes. Abbreviation: GMT, Gata4, Mef2c, and Tbx5. 
The induced cardiomyocytes had striated $\alpha$-actinin structure and expressed six characteristic markers of cardiomyocytes: $\alpha-$ MHC, Actc1, Actn2, Gja1, Hand2, and Tnnt2.

\section{Experimental section \\ Materials}

pcDNA $^{\mathrm{TM}} 6 / \mathrm{V} 5$-His B, Iscove's Modified Dulbecco's Medium (IMDM), Dulbecco's Modified Eagle's Medium (DMEM), fetal bovine serum (FBS), non-essential amino acids (NEAA), sodium pyruvate, GlutaMax, penicillinstreptomycin, medium M199, OptiMEM I reduced-serum medium, lipofectamine 2000, Ulysis Alexa Fluor 594 nucleic acid labeling kit, 4',6-diamidino-2-phenylindole (DAPI) for nucleus staining, and lipofectamine RNAiMax were purchased from Thermo Fisher Scientific (Waltham, MA, USA). NEB 5-alpha competent Escherichia coli and Antarctic phosphatase were obtained from New England Biolabs (Ipswich, MA, USA). 3'-O-Me-m ${ }^{7} \mathrm{G}\left(5^{\prime}\right) \mathrm{ppp}\left(5^{\prime}\right) \mathrm{G}$ RNA cap analog, 5-methylcytidine-5' -triphosphate (Me-CTP), pseudouridine5 'triphosphate (Pseudo-UTP), and enhanced green fluorescence protein (eGFP) mRNA were purchased from TriLink Biotechnologies (San Diego, CA, USA). Gelatin 0.1\% solution and branched polyethylenimine (bPEI, $10 \mathrm{kDa}$ ) were purchased from Sigma-Aldrich Co. (St Louis, MO, USA). CRPPR-R9 (CRPPRGGGGRRRRRRRRR) and its control sequence, CRRPP-R9 (CRRPPGGGGRRRRRRRRR), were synthesized by Peptide 2.0 (Chantilly, VA, USA). Plasmid Midi Kit and QIAquick spin columns were purchased from Qiagen NV (Venlo, the Netherlands). Stemfect was obtained from Stemgent (Cambridge, MA, USA). TransIT-X2 and TransIT-mRNA were purchased from Mirus Bio LLC (Madison, WI, USA). Micro Bio-Spin P-6 column, Aurum Total RNA Mini kit, iScript cDNA synthesis kit, and SsoAdvanced Universal SYBR Green Supermix were obtained from Bio-Rad Laboratories Inc. (Hercules, CA, USA).

\section{Cardiac fibroblast isolation and culture}

Neonatal cardiac fibroblasts were isolated as previously described following a conventional neonatal cardiac fibroblast isolation method. ${ }^{20}$ GFP-negative cardiac fibroblasts from genetically modified neonatal mice that express GFP by the $\alpha$-MHC promoter ( $\alpha-M H C$-GFP) were sorted from migrated cells using MoFlo fluorescence-activated cell sorting (FACS; Beckman Coulter, Brea, CA, USA). GFP-/ Thy $1+$ cells were sorted and cultured for direct cardiac reprogramming experiments. ${ }^{20}$ Growth culture media was IMDM containing 20\% FBS, 1\% NEAA, 1\% sodium pyruvate, $1 \%$ GlutaMAX, and 5\% penicillin-streptomycin.
Fresh cardiac fibroblasts, within 7 days from the isolation, were used for experiments. Cardiomyocyte medium that was given to cardiac fibroblasts after transfection was composed of DMEM and M199, 10\% FBS, 1\% NEAA, 1\% sodium pyruvate, and 1\% GlutaMAX. All cells were cultured at $37^{\circ} \mathrm{C}$ and $5 \% \mathrm{CO}_{2}$.

\section{Primary cardiac fibroblast transfection with conventional transfection reagents}

We tested various commercial transfection reagents including lipofectamine 2000, lipofectamine RNAiMAX, Stemfect, TransIT-X2, TransIT-mRNA (Mirus Bio LLC), and also polycationic polymers such as bPEI and PEG-polylysine (22 ethylene glycol units, 50 lysine units; Alamanda Polymers Inc., Huntsville, AL, USA). Transfection was optimized by standard procedures or the manufacturer's protocol. eGFP mRNA transfection was conducted overnight, washed with phosphate buffered saline (PBS), and observed with a fluorescence microscope. eGFP-expressing cells with optimized transfection conditions were analyzed using flow cytometry (Guava easyCyte; EMD Millipore, Billerica, MA, USA).

\section{Cellular uptake of Alexa594-mRNA via CRPPR-R9 in cardiac fibroblasts}

Alexa594 labeling was conducted on $10 \mu \mathrm{g}$ of eGFP mRNA with Ulysis Alexa Fluor 594 nucleic acid labeling kit (Thermo Fisher Scientific) following the manufacturer's manual. Excess unreacted dyes were removed using a Micro Bio-Spin P-6 column (Bio-rad Laboratories Inc.). Cardiac fibroblasts $\left(2 \times 10^{4}\right.$ cells/well) were cultured on Millicell EZ slide (EMD Millipore) overnight. The Alexa594-labeled mRNA $(500 \mathrm{ng} / \mu \mathrm{L}, 1 \mu \mathrm{L})$ was prepared with CRPPR-R9 (PBS, $1 \mu \mathrm{g} / \mu \mathrm{L}, 3.8 \mu \mathrm{L}$ ) at a molar charge ratio of 20 in OptiMEM. The cardiac fibroblasts were transfected with mRNA/CRPPR-R9 complex: Alexa594-labeled mRNA (500 ng), CRPPR-R9 (3.8 $\mu \mathrm{g})$, and only Alexa594-labeled mRNA control (500 ng). After 30 minutes incubation, the culture medium was removed and cells were washed with PBS three times. Cardiac fibroblasts were fixed in $4 \%$ paraformaldehyde (Santa Cruz Biotechnology, Inc., Dallas, TX, USA) and the nucleus was stained with DAPI. The fixed cells were observed by swept field confocal microscopy (SFCM; Prairie Technology, Middleton, WI, USA).

\section{mRNA transfection to cardiac fibroblasts}

Cardiac fibroblasts $\left(5 \times 10^{4}\right.$ cells per well) were seeded in a 24-well plate 1 day before transfection. eGFP mRNA $(0.5 \mu \mathrm{g})$ was added to $50 \mu \mathrm{L}$ of OptiMEM in a $1.5 \mathrm{~mL}$ Eppendorf tube. 
The calculated amount of CRPPR-R9 (0.95 $\mu \mathrm{g}, 3.8 \mu \mathrm{g}$, and $9.5 \mu \mathrm{g}$ resulting in 5,20 , and 50 molar charge ratio) or scramble sequence CRRPP-R9 (3.8 $\mu \mathrm{g}, 20$ molar charge ratio) was added to the eGFP mRNA solution, and was incubated at room temperature for 15 minutes. Next, $1 \mu \mathrm{L}$ of lipofectamine 2000 in $50 \mu \mathrm{L}$ OptiMEM was added to the solution and further incubated for 15 minutes. We describe sequential addition of CRPPR-R9 and lipofectamine, but simultaneous mixing of all three components is also a viable approach. After washing cells with warm PBS, the prepared eGFP mRNA complexes $(100 \mu \mathrm{L})$ were transfected to the cells in $500 \mu \mathrm{L}$ OptiMEM. The control cells were treated with only OptiMEM buffer or lipofectamine without peptides in the same ratio of lipofectamine to eGFP mRNA. Only CRPPR-R9 cells were treated with eGFP mRNA $(0.5 \mu \mathrm{g})$ and CRPPR-R9 $(3.8 \mu \mathrm{g})$ mixture. The cells were incubated overnight and washed with PBS, followed by the addition of fresh growth media. At 24 hours post-transfection, fluorescence microscopic observations and flow cytometry analyses were conducted. Transfection efficiency was quantified based on the percentage of eGFP+ cardiac fibroblasts and mean eGFP fluorescence intensity of individual cells from the whole cell population after transfection was quantified by InCyte software (EMD Millipore).

\section{Transfection efficiency and cytotoxicity optimization of CRPPR-R9/lipofectamine transfection}

Cardiac fibroblasts $\left(5 \times 10^{4}\right.$ cells/well $)$ were seeded in a 24 -well plate 1 day prior to transfection. Gata 4 mRNA $(0.5 \mu$ g per well) was mixed with lipofectamine ( $1 \mu \mathrm{L}$ per well) or C-Lipo (3.8 $\mu \mathrm{g}$ of CRPPR-R9 and $0.25 \mu \mathrm{L}$ of lipofectamine per well), and was transfected for 4 hours in OptiMEM and further incubated in growth DMEM media for 20 hours. The same transfection was conducted daily for 1 week. On days 4, 7, and 14 of transfection, cells were washed with PBS and cell viability was measured with Cell Counting Kit-8. Cell viability was quantified with absorbance at $450 \mathrm{~nm}$ and normalized by the cell viability of the control cells without transfection. For transfection efficiency and mean eGFP expression analysis, transfection with the same formulation as described earlier, was conducted overnight and washed with PBS, followed by the addition of fresh growth media. At 24 hours post-transfection, flow cytometry analyses were conducted.

\section{Construction of in vitro transcription templates}

The oligonucleotide sequences of Mef $2 \mathrm{c}$ and Tbx 5 were procured from pMX vectors as previously reported. ${ }^{2}$ A pcMV6 vector containing Gata4 (MG227022) was purchased from Origene Technologies, Inc. (Rockville, MD, USA). BamHI and NotI restriction enzymes were used to cut Mef2c and Tbx5 genes from the pMX vector, and the Gata4 pcMV6 vector was digested with BamHI and XhoI. The resulting digested genes were cloned in pcDNA ${ }^{\mathrm{TM}} 6 / \mathrm{V} 5$-His $\mathrm{B}$, ligated with T4 DNA ligase (New England Biolabs), and then transformed into DH5 $\alpha$ by heat-shock treatments. Each GMT pcDNA plasmid was isolated by Plasmid Midi Kit (Qiagen NV) following the manufacturer's manual.

\section{Synthesis of modified mRNAs}

Modified mRNAs that are made of bases with modification for efficient translation and stability were synthesized with the MEGAscript T7 transcription kit (Life Technologies, Grand Island, NY). Individual GMT pcDNA plasmids were linearized by adding XhoI restriction enzyme and incubating for 1 hour at $37^{\circ} \mathrm{C}$. The resulting linearized plasmids were then purified with QIAquick spin columns (Qiagen NV). Four micrograms of purified linear plasmids for each $40 \mu \mathrm{L}$ reaction were prepared. Adenosine triphosphate and guanosine triphosphate from the kit were used. The modified bases, $3^{\prime}-0$ Me-m $\mathrm{m}^{7} \mathrm{G}\left(5^{\prime}\right) \mathrm{ppp}\left(5^{\prime}\right) \mathrm{G}$ ARCA cap analog, 5-methylcytidine triphosphate, and pseudouridine triphosphate (TriLink Biotechnologies, San Diego, CA, USA), were also mixed with the two triphosphates from the kit. Final nucleotide reaction concentrations were $6 \mathrm{mM}$ for the cap analog, $1.5 \mathrm{mM}$ for guanosine triphosphate, and $7.5 \mathrm{mM}$ for the other nucleotides. mRNA synthesis mixtures were incubated for 6 hours at $37^{\circ} \mathrm{C}$, followed by Turbo DNase treatment for 30 minutes. Poly(A) tailing (Life Technologies) was conducted. Antarctic phosphatase (New England Biolabs) was added and the mixture was incubated for 30 minutes at $37^{\circ} \mathrm{C}$ to remove residual 5 'triphosphates. RNA was purified with MEGAclear spin columns as described in the manufacturer's manual. RNA reprogramming cocktails were prepared by pooling individual RNA stocks with the ratio suitable for each experiment and a $100 \mathrm{ng} / \mu \mathrm{L}$ blend was prepared and frozen at $-80^{\circ} \mathrm{C}$ until transfections were conducted.

\section{Direct cardiac reprogramming using GMT mRNA transfection via C-Lipo}

Cardiac fibroblasts $\left(3 \times 10^{5}\right.$ cells/well $)$ were prepared in gelatin-coated six-well plates and grown until cell density reached $70 \%$ confluency. Growth media was removed and the cells were PBS washed before transfection. GMT mRNA $(1.2 \mu \mathrm{g})$ and CRPPR-R9 $(9.12 \mu \mathrm{g})$ were mixed in $50 \mu \mathrm{L}$ of OptiMEM for 10 minutes at room temperature. Lipofectamine 
$(0.6 \mu \mathrm{L}$ in $50 \mu \mathrm{L}$ of OptiMEM) was added to the complex and incubated for an additional 15 minutes at room temperature. Transfection mix in $700 \mu \mathrm{L}$ of OptiMEM was added to cells and incubated for 4 hours. The cells were washed with PBS and further incubated in growth media. Daily transfections were conducted for 14 consecutive days and cell morphology was monitored. $\alpha$-MHC-GFP-transfected cardiac fibroblasts were observed with fluorescence microscopy on day 7 and images were taken and processed with QCapture Pro 6 (QImaging, Surrey, BC, Canada). GFP+ population percentage was quantified using flow cytometry and InCyte software at day 14 of transfection.

\section{Immunohistochemistry staining for sarcomeric protein, $\alpha$-actinin}

After 14 times of daily transfection of neonatal cardiac fibroblasts without $\alpha-M H C$-GFP reporter, the culture medium was removed and cells were washed with PBS three times. Induced cardiomyocytes were fixed in 4\% paraformaldehyde (Santa Cruz Biotechnology, Inc.). After washing, blocking buffer containing 5\% goat serum was given for 30 minutes at room temperature. $\alpha$-actinin primary antibody (sc-15335; Santa Cruz Biotechnology, Inc.) was diluted 1:200 in blocking buffer and incubated overnight at $4^{\circ} \mathrm{C}$. After washing, secondary goat anti-rabbit IgG-CFL 488 antibody (sc-362262; Santa Cruz Biotechnology, Inc.) was added and incubated for 1 hour at room temperature. After nucleus staining with DAPI, the stained cells were observed by SFCM (Prairie Technology).

\section{Cardiomyocyte marker gene expression analysis}

After 14 times of daily transfection, the transfected cells were cultured an additional 4 days from the last transfection in a cardiomyocyte medium. On day 18 from the initial transfection, total cells were lysed and mRNAs were extracted using Aurum Total RNA Mini Kit (Bio-rad Laboratories Inc.). cDNAs were prepared via iScript cDNA synthesis kit (Biorad Laboratories Inc.) and real-time polymerase chain reaction (RT-PCR) reactions were performed with SsoAdvanced Universal SYBR Green Supermix (Bio-rad Laboratories Inc.). Primers are listed in Table S1. CFX Real-Time PCR system (Bio-Rad Laboratories Inc.) was used and data were analyzed by iQ5 software (Bio-Rad Laboratories Inc.). GAPDH was used for loading control and normalization.

\section{Stoichiometric analysis}

Different ratios of the Gata4, Mef2c, and Tbx5 mRNAs were used: an equal ratio of GMT mRNAs (1:1:1), a three-fold excess of Gata4 to Mef2c and Tbx5 (3:1:1), and a three-fold excess of Tbx 5 to Gata 4 and Mef2c (1:1:3). The total mRNA amount was $1.2 \mu \mathrm{g}$ for all samples and the same transfection method as described above was conducted. After 10 times of daily transfection, the transfected cells were cultured an additional 4 days from the last transfection. On day 14 from the initial transfection, mRNA was extracted and qRT-PCR was conducted as described previously.

\section{Statistical analysis}

$P$-values were determined by Student's $t$-test and one-way ANOVA using GraphPad Prism (GraphPad Software, Inc., La Jolla, CA, USA). $P$-values of less than 0.05 and 0.01 are indicated as $*$ and $* *$, respectively.

\section{Results and discussion Conventional transfection reagents are not effective or are toxic to cardiac fibroblasts}

mRNA transfection to primary cardiac cells such as cardiac fibroblasts has been challenging. ${ }^{15,16}$ We tested seven different transfection reagents on cardiac fibroblasts with eGFP mRNA, and all of the reagents had very low transfection efficiency, except for lipofectamine 2000 (described henceforth as lipofectamine) which had a $20 \%$ transfection efficiency (Figure S1). However, lipofectamine caused severe cell damage after 1 week of daily transfection, which is too short a period to induce direct cardiac reprogramming. Therefore, we designed a peptide-assisted transfection system that targets cardiac cells to improve the mRNA transfection efficiency of lipofectamine and also lower its cytotoxicity.

\section{CRPPR-R9 enhances the uptake of mRNA in cardiac fibroblasts}

Peptide-assisted mRNA transfection is a promising way to overcome the intrinsic nature of lipofectamine that causes low efficiency and high toxicity to primary cells. We hypothesized that peptide targeting with CRPPR-R9 would improve the cardiac fibroblast transfection efficiency of lipofectamine by enhancing endocytosis, and would enable direct cardiac reprogramming with mRNAs. We investigated if CRPPR-R9 could enhance the uptake of Alexa594-labeled eGFP mRNA. CRPPR-R9 was complexed with Alexa594labeled eGFP mRNA at a molar charge ratio of 20:1. The formation of the CRPPR-R9/mRNA complex was confirmed with an electrophoretic mobility shift assay (Figure S2). The CRPPR-R9/mRNA complex was incubated with cardiac 
fibroblasts for 30 minutes, and analyzed by fluorescence microscopy. Figure 2A demonstrates that the CRPPR-R9/ mRNA complex is robustly internalized, while cardiac fibroblasts treated with Alexa594-labeled mRNA alone exhibited minimal fluorescence.

\section{CRPPR-R9 enhances the mRNA transfection efficiency of lipofectamine}

To investigate if CRPPR-R9 can form complexes with mRNA and lipofectamine, the CRPPR-R9/eGFP mRNA complex was mixed with lipofectamine. The complexes
A

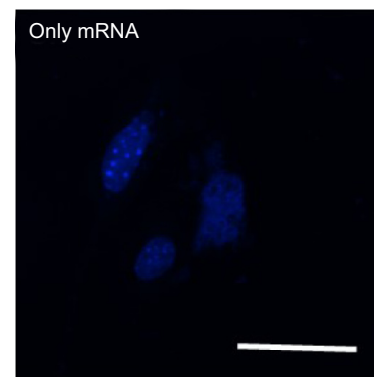

B

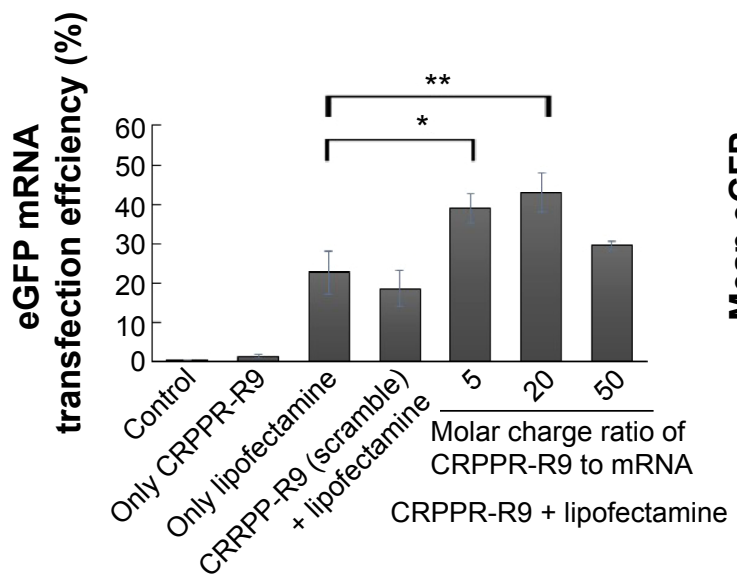

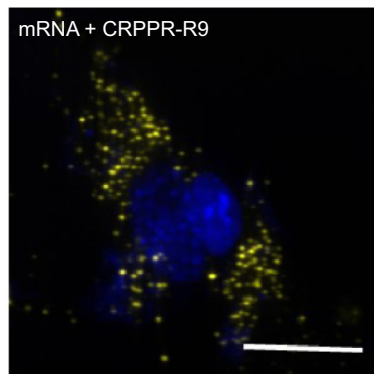

C

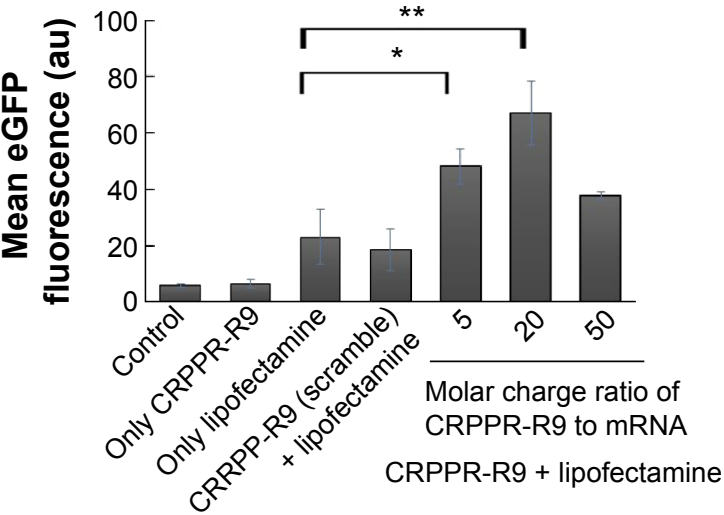

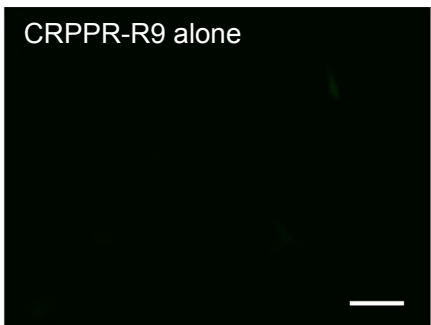

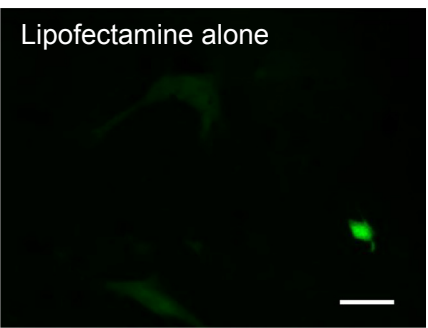

Scrambled CRRPP-R9 +
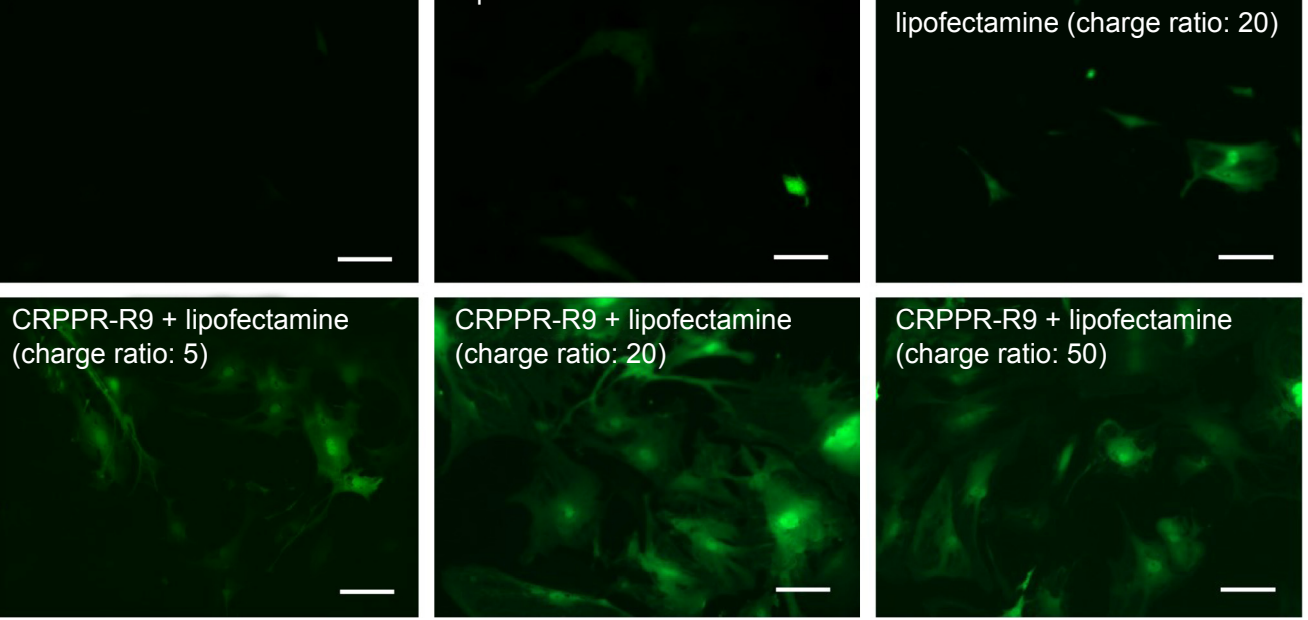

Figure 2 CRPPR-R9 facilitates mRNA delivery in cardiac fibroblasts and CRPPR-R9/lipofectamine improves mRNA transfection efficiency.

Notes: (A) Confocal images of cardiac fibroblasts treated with Alexa594-labeled mRNA/CRPPR-R9 complexes. mRNA and mRNA/CRPPR-R9 complexes were transfected into cardiac fibroblasts for 30 minutes. CRPPR-R9 induces facilitated mRNA uptake in cardiac fibroblasts. Alexa594-labeled mRNA and nucleus are shown in yellow and blue, respectively. Scale bar is $10 \mu \mathrm{m}$. (B) Transfection efficiency of eGFP mRNA on cardiac fibroblasts transfected by various combinations of CRPPR-R9 and lipofectamine and quantified by flow cytometry. CRPPR-R9 with lipofectamine increases eGFP mRNA transfection efficiency. (C) Mean eGFP fluorescence of cardiac fibroblasts quantified by flow cytometry. Mean eGFP fluorescence increases as transfection efficiency increases in CRPPR-R9 and lipofectamine transfection. The results represent the mean \pm SE, $\mathrm{n}=4$. $* \mathrm{P}<0.05$; $* * P<0.01$. (D) Images of eGFP fluorescence in cardiac fibroblasts with eGFP mRNA. Scale bar is $10 \mu \mathrm{m}$.

Abbreviations: eGFP, enhanced green fluorescence protein; SE, standard error. 
formed from CRPPR-R9/mRNA/lipofectamine were centrifuged, isolated, and analyzed by gel electrophoresis for the presence of CRPPR-R9 and mRNA. Figure S3 shows the presence of CRPPR-R9 in the CRPPR-R9/mRNA/ lipofectamine complex. We investigated if CRPPR-R9 can improve the mRNA transfection efficiency of lipofectamine on cardiac fibroblasts. The CRPPR-R9/mRNA/ lipofectamine complex was treated to cardiac fibroblasts, and eGFP expression was determined by flow cytometry and fluorescence microscopy. Figure $2 \mathrm{~B}$ demonstrates that CRPPR-R9/lipofectamine dramatically increases the eGFP mRNA transfection efficiency of lipofectamine. For example, CRPPR-R9/lipofectamine (charge ratio of 20) had an mRNA transfection efficiency of $44 \%$, which was about two-fold higher than lipofectamine alone. In addition, the mean eGFP fluorescence in the CRPPR-R9/lipofectamine-transfected cardiac fibroblast cell was also increased by about two-fold in comparison to lipofectamine (Figure 2C). CRRPP-R9, a scrambled sequence with the same charge profiles as CRPPR-R9, was mixed with eGFP mRNA and lipofectamine (1:20), and eGFP expression in cardiac fibroblasts showed no improvement compared to lipofectamine alone. The results suggest that the enhanced transfection is due to the CRPPR sequence-specific interaction. Figure 2D shows fluorescence images of transfected cardiac fibroblasts that demonstrate improved lipofectamine transfection via CRPPR-R9. Additional transfection experiments in HeLa cells, a cervical cancer cell-line, show that CRPPR-R9 does not improve, and instead hampers, the transfection efficiency of lipofectamine in HeLa cells (Figure S4). Moreover, CRPPR-R9 alone had no toxicity to cardiac fibroblasts under these transfection conditions (Figure S5).

\section{CRPPR-R9 allows comparable levels of lipofectamine transfection with low toxicity}

A key challenge in direct reprogramming with mRNA is the need for consecutive transfections for weeks. Transfection reagents such as lipofectamine generate significant toxicity if used repeatedly. Therefore, we investigated if C-Lipo with $75 \%$ less lipofectamine $(0.5 \mu \mathrm{L}$ of lipofectamine with $7 \mu \mathrm{g}$ of CRPPR-R9 peptide per $1 \mu \mathrm{g}$ of mRNA) could transfect cells consecutively for 2 weeks with minimal toxicity by reducing the amount of lipofectamine given to the cells. Cardiac fibroblasts were treated with mRNA and either lipofectamine (Lipo, $2 \mu \mathrm{L}$ lipofectamine per $1 \mu \mathrm{g}$ mRNA) or C-Lipo, using $25 \%$ as much lipofectamine, daily for 1 week; the cell viability and transfection efficiency were measured. Figure 3A demonstrates that C-Lipo has lower toxicity than Lipo. C-Lipo caused a $25 \%$ cell viability drop after 1 week of transfection, whereas Lipo caused a 90\% cell viability drop, and the viability difference between the two transfection reagents became more significant in week 2 . The higher cell viability of C-Lipo transfection in comparison to lipofectamine is mainly due to the reduced amount of lipofectamine (Figure S6). In addition, C-Lipo had a transfection efficiency that was similar to Lipo and had a higher mean eGFP expression level per cell (Figures 3B and C). These results show that $\mathrm{C}$-Lipo can efficiently transfect cardiac fibroblasts with low cytotoxicity.
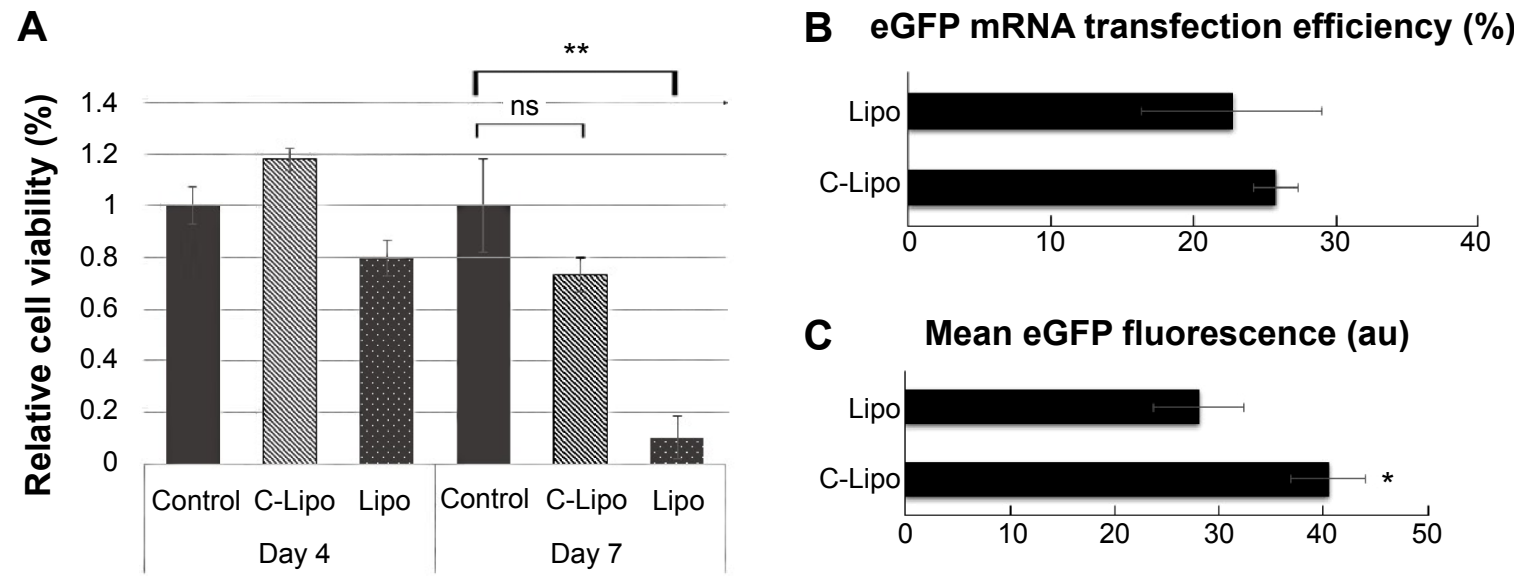

Figure 3 C-Lipo with reduced lipofectamine amount transfects cardiac fibroblasts efficiently without significant toxicity.

Notes: (A) Cell viability of C-Lipo ( $0.5 \mu \mathrm{L}$ of lipofectamine with $7 \mu \mathrm{g}$ of CRPPR-R9 peptide per I $\mu \mathrm{g}$ mRNA) after daily transfections. Cardiac fibroblasts were transfected with C-Lipo and Lipo daily for I week and cell viabilities were measured on days 4 and 7. C-Lipo allows mRNA transfection in cardiac fibroblasts without significant toxicity for I week compared to Lipo, which causes significant toxicity. (B) and (C) Flow cytometry analyses of cardiac fibroblasts treated with C-Lipo and Lipo. C-Lipo shows comparable levels of transfection efficiency and higher mean eGFP fluorescence compared to Lipo. The results represent the mean $\pm S E$, $n=5$. $* P<0.05$; $* * P<0.0$ I. Abbreviations: eGFP, enhanced green fluorescence protein; SE, standard error; ns, not significant. 


\section{GFP expression in $\alpha$-MHC-GFP cardiac} fibroblasts after GMT mRNA transfection via C-Lipo

We investigated if transfection of GMT mRNAs with C-Lipo could induce the expression of $\alpha-M H C$, a cardiomyocyte marker gene, from cardiac fibroblasts. Cardiac fibroblasts that have the GFP gene driven by the $\alpha-M H C$ promoter were isolated from neonatal transgenic mice. Fresh $\alpha$-MHC-GFP cardiac fibroblasts were transfected with GMT mRNAs via
C-Lipo every day for 7 days. Figure 4A demonstrates that GMT mRNA transfection with C-Lipo does induce $\alpha$-MHC expression in $0.5 \%$ of transfected cardiac fibroblasts. GFP expression from GMT mRNA-transfected cardiac fibroblasts was less compared to the reported result of retroviral transduction $(17 \%))^{2,3} \mathrm{GFP}+$ cells first appeared at day 7 from the initial transfection. GFP+ cells consistently expressed GFP for 20 days of culture until we discontinued observation.
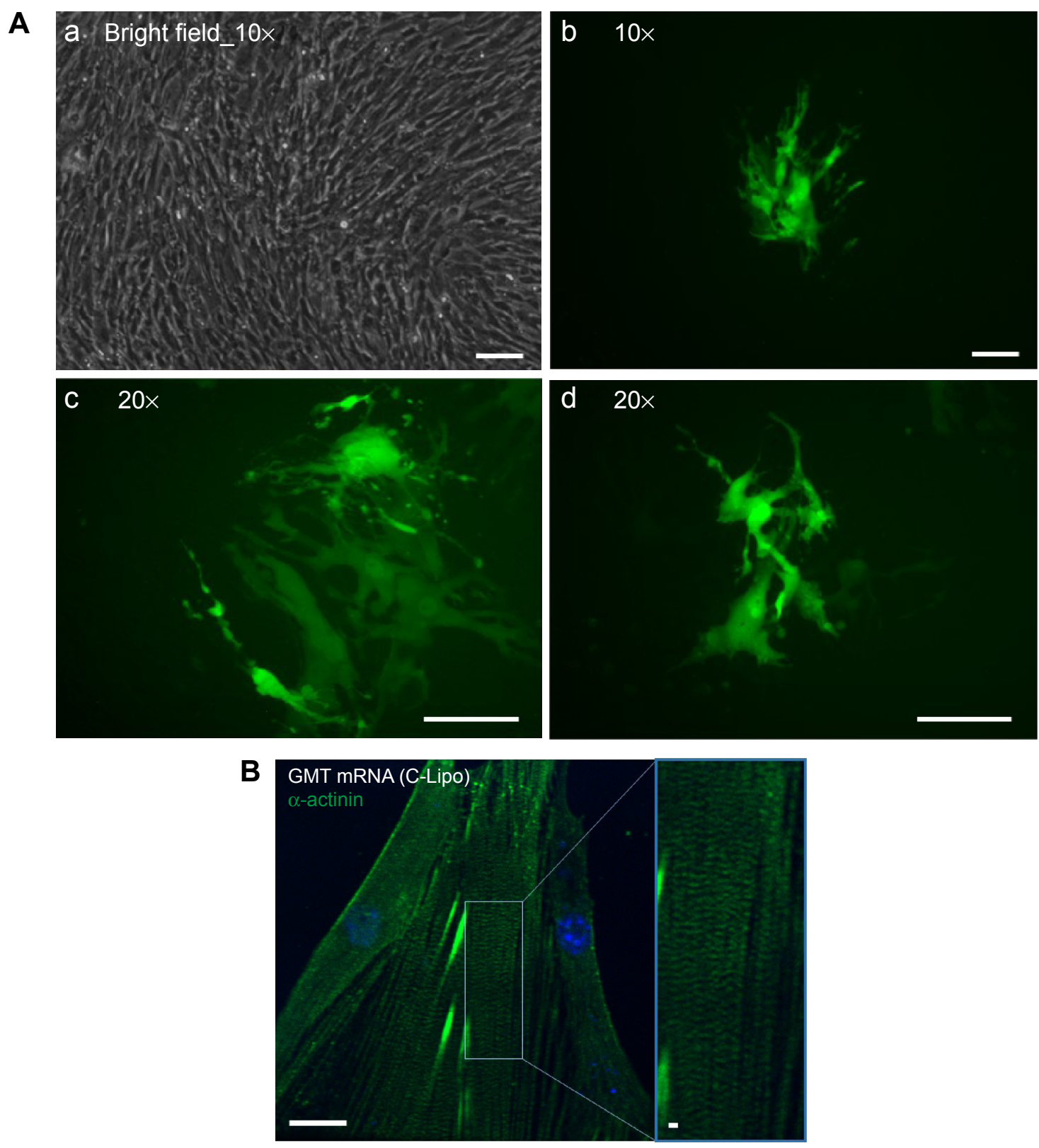

Figure 4 Transfection of GMT mRNAs on cardiac fibroblasts induces expression of cardiomyocyte marker genes and striated cardiac muscle structure.

Notes: (A) Fluorescence images of $\alpha$-MHC promoter-driven GFP expression from GMT mRNA transfected cardiac fibroblasts. $\alpha$-MHC-GFP cardiac fibroblasts were transfected daily with GMT mRNAs for 7 days and observed with fluorescence microscope. (a, b) Both bright field and green fluorescence images were taken with $10 \times$ magnification. (c, d) $20 \times$ magnification images were taken from two additional regions. Scale bar is $10 \mu \mathrm{m}$. (B) Immunohistochemistry staining of $\alpha$-actinin in GMT mRNA/CLipo transfected cardiac fibroblasts. Inducted cardiomyocyte-like cells express $\alpha$-actinin and have striated cardiac muscle structure. Scale bar is $2 \mu \mathrm{m}$. Abbreviations: GFP, green fluorescence protein; GMT, Gata4, Mef2c, and Tbx5. 


\section{Cardiomyocyte marker genes are upregulated in cardiac fibroblasts} transfected with GMT mRNA via C-Lipo

We investigated if transfection of cardiac fibroblasts for 14 days with GMT mRNA/C-Lipo could induce the characteristics of cardiomyocytes using immunostaining and RT-PCR. Figure 4B demonstrates that $\alpha$-actinin is expressed in the induced cardiomyocyte-like cells after GMT mRNA/C-Lipo transfection and striated muscle structure is observed with $\alpha$-actinin staining. ${ }^{20}$ Additionally, Figure 5 demonstrates that 14 days of transfection with GMT mRNA/C-Lipo induced a significant increase in the levels of many cardiomyocyte marker genes. For example, the cardiomyocyte marker genes that are important in cardiomyocyte development and function: Actc1, Actn2, Gja1, Hand2, and Tnnt2, were significantly upregulated in comparison to their levels in the control cardiac fibroblasts without transfections. These results further demonstrate that the transfection of GMT mRNA/C-Lipo induces cardiomyocyte marker genes from cardiac fibroblasts, suggesting direct reprogramming towards the cardiomyocyte lineage. The expression profile of the marker genes in the cells transfected with only lipofectamine was unavailable for
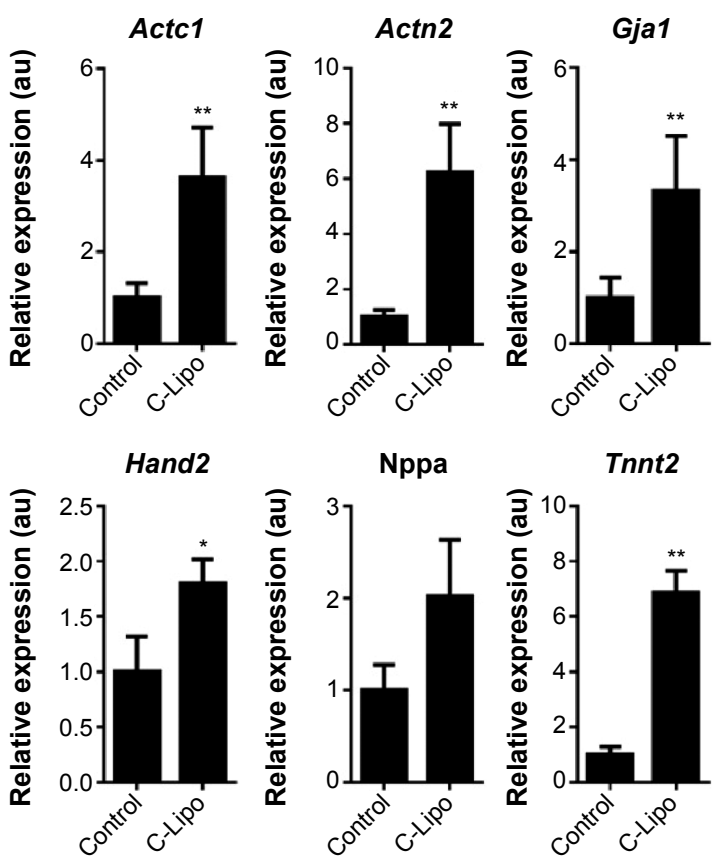

Figure 5 Transfection of GMT mRNAs via C-Lipo induces expression of cardiomyocyte marker genes from transfected cardiac fibroblasts.

Notes: Quantification of the relative expression of cardiomyocyte marker genes after 2 weeks of transfection. GMT mRNA transfection via C-Lipo drives cardiac fibroblasts to express cardiomyocyte marker genes: Actcl, Actn2, Gjal, Hand2, and Tnnt2. The results represent the mean $\pm S E, n=3$. $* P<0.05 ; * * P<0.01$. Abbreviations: GMT, Gata4, Mef2c, and Tbx5; SE, standard error. further studies due to significant cell death after transfection for 1 week. The results of $\alpha$-MHC-GFP expression, striated cardiac structure from $\alpha$-actinin staining, and cardiomyocyte marker gene overexpression collectively support the initiation of direct cardiac reprogramming toward cardiomyocyte-like cells.

\section{Stoichiometric ratio of GMT mRNAs affects the expression pattern of induced cardiomyocyte-like cells}

mRNA transfection for direct reprogramming has the advantage of precisely controlling the stoichiometry of reprogramming factors. Understanding the optimal stoichiometric ratio of reprogramming factors is a key step toward addressing the significant challenges of improving the reprogramming efficiency and maturity of reprogrammed cells. ${ }^{21}$ In order to understand how the ratio of reprogramming factors affects the direct reprogramming process, a stoichiometric study was conducted with three ratios of mRNAs: equal molar ratio of Gata4, Mef2c, and Tbx 5 (1:1:1), three-fold molar excess of Gata4 to Mef2c and Tbx5 (3:1:1), and three-fold molar excess of Tbx 5 to Gata 4 and Mef2c (1:1:3). The ratios of reprogramming factors were decided based on the human induced pluripotent stem (hiPS) cell result that reprogramming was efficient when the Oct4 gene was expressed three times more than other reprogramming factors. ${ }^{22}$ Figure 6 demonstrates that stoichiometry plays a major role in controlling cardiomyocyte marker gene expression patterns. For example, excess Tbx 5 (1:1:3) induced higher expression of Gjal and Hand2, and excess Gata4 (3:1:1) increased the expression of Nppa and Tnnt2. High levels of Nppa expression were observed in cells transfected with excess Gata4 mRNA (3:1:1), which can be explained by a direct transcriptional regulation of Nppa by Gata $4 .{ }^{23}$ Also, the fact that excess Tbx 5 mRNA (1:1:3) induces the overexpression of Gjal, a gap junction alpha-1 protein, which is a key player in the synchronized contraction of the heart, implies the importance of Tbx 5 in functionality of induced cardiomyocytes. ${ }^{24}$ Our stoichiometric study demonstrated that the expression of cardiomyocyte marker genes is dependent on the ratio of GMT mRNAs. This is the first demonstration of the effect of stoichiometry of reprogramming factors in direct cardiac reprogramming to the best of our knowledge. mRNA has an advantage of precise control on stoichiometry and mRNA stoichiometric studies have a high potential to improve the efficiency of direct reprogramming and the functionality of induced cardiomyocytes. 

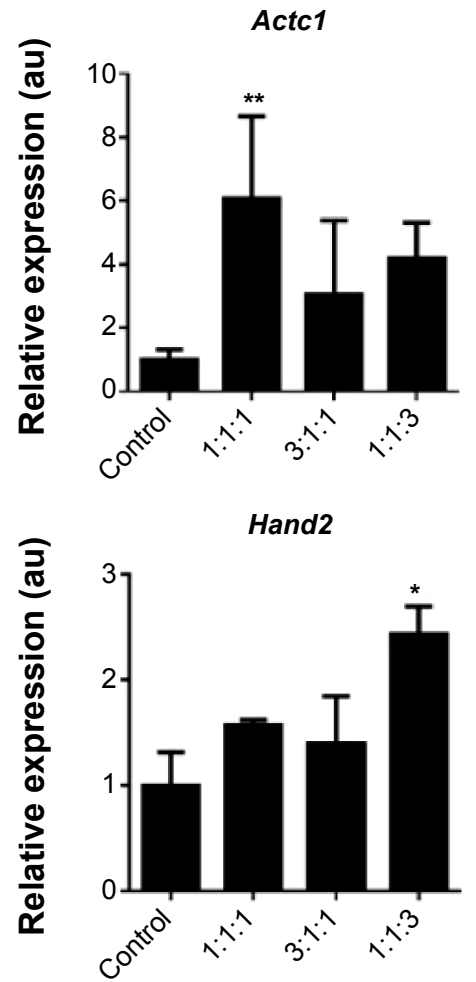
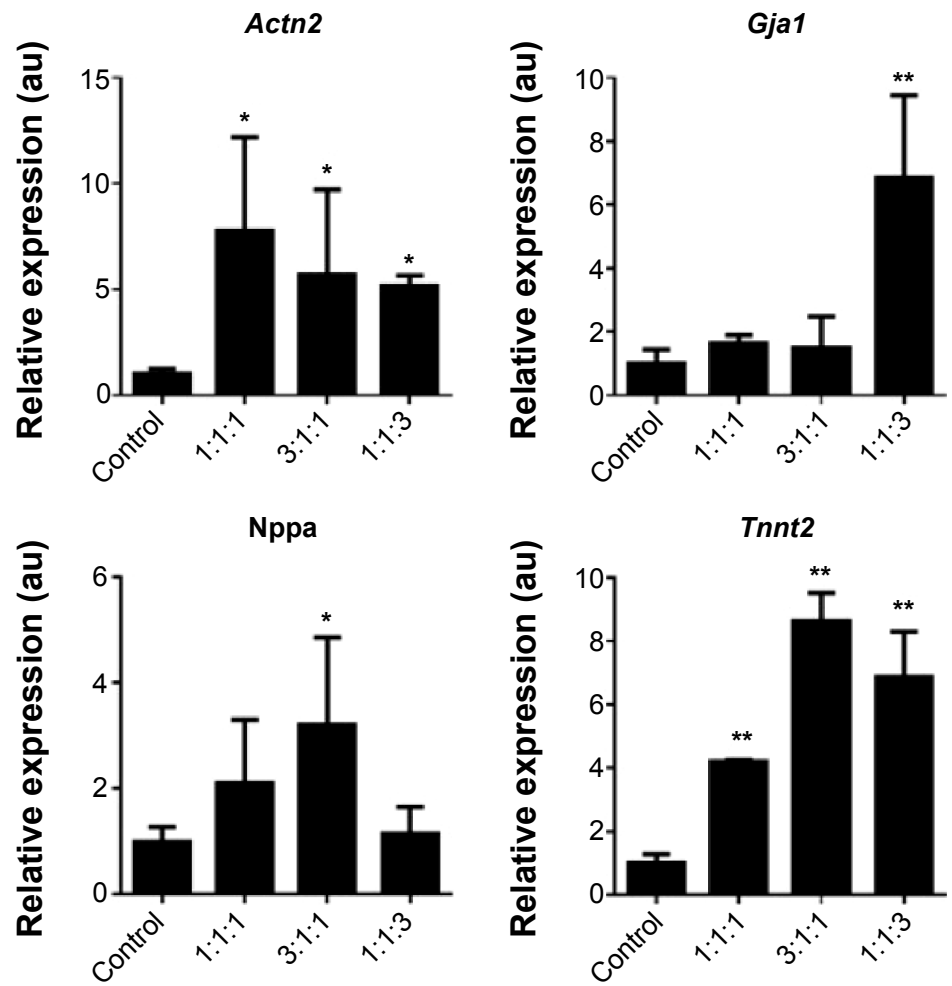

Figure 6 Stoichiometric ratio of GMT mRNAs influences cardiomyocyte marker gene expression patterns.

Notes: Quantification of the relative expression of cardiomyocyte marker genes in cardiac fibroblasts transfected with various stoichiometric ratios of GMT mRNAs. Gene expression levels of Actcl, Actn2, Gja I, Hand2, Nppa, and Tnnt2 were affected by the stoichiometric ratio of GMT mRNAs. The results represent the mean \pm SE, $\mathrm{n}=3$. $* \mathrm{P}<0.05$; $* * P<0.01$; statistical significance was tested between the control and each sample. Abbreviations: GMT, Gata4, Mef2c, and Tbx5; SE, standard error.

\section{Conclusion}

In this study, we present a peptide-assisted mRNA transfection method that can induce the direct reprogramming of cultured mouse cardiac fibroblasts towards cardiomyocytelike cells with moderate efficiency. The C-Lipo significantly reduces cytotoxicity and improves cell viability during multiple mRNA transfections, in comparison to lipofectamine transfection. GMT mRNA transfection via C-Lipo induced significant overexpression of cardiomyocyte marker genes in cultured mouse cardiac fibroblasts. This study demonstrates that mRNA can be utilized for direct cardiac reprogramming and is useful for stoichiometric control of reprogramming factors. Furthermore, our research may provide groundwork for the development of in vivo mRNA delivery vehicles to the infarcted heart.

\section{Acknowledgments}

This work was supported by the grants from NIH, U01 268201000043C-0-0-1, R56 AI107116-01, and RO1 AI088023-03. In addition, this work was supported by the WM Keck Foundation, based in Los Angeles, USA. We thank M West in the Berkeley Stem Cell Center and Mr H Nolla in the FACS facility for technical assistance. We thank
Mr Junren Sia and Dr Song Li for generous support. We thank M Webb for advice and proofreading.

\section{Disclosure}

The authors report no conflicts of interest in this work.

\section{References}

1. Qian L, Huang Y, Spencer C, et al. In vivo reprogramming of murine cardiac fibroblasts into induced cardiomyocytes. Nature. 2012; 485(7400):593-598.

2. Ieda M, Fu JD, Delgado-Olguin P, et al. Direct reprogramming of fibroblasts into functional cardiomyocytes by defined factors. Cell. 2010; 142(3):375-386.

3. Song K, Nam YJ, Luo X, et al. Heart repair by reprogramming nonmyocytes with cardiac transcription factors. Nature. 2012;485(7400): 599-604.

4. Warren L, Manos PD, Ahfeldt T, et al. Highly efficient reprogramming to pluripotency and directed differentiation of human cells with synthetic modified mRNA. Cell Stem Cell. 2010;7(5):618-630.

5. Zhou H, Wu S, Joo J, et al. Generation of induced pluripotent stem cells using recombinant proteins. Cell Stem Cell. 2009;4(5):381-384.

6. Adler AF, Grigsby CL, Kulangara K, Wang H, Yasuda R, Leong KW. Nonviral direct conversion of primary mouse embryonic fibroblasts to neuronal cells. Mol Ther Nucleic Acids. 2012;1:e32.

7. Warren L, Ni Y, Wang J, Guo X. Feeder-free derivation of human induced pluripotent stem cells with messenger RNA. Sci Rep. 2012;2:657.

8. Okita K, Nakagawa M, Hyenjong H, Ichisaka T, Yamanaka S. Generation of mouse induced pluripotent stem cells without viral vectors. Science. 2008;322(5903):949-953. 
9. Mandal PK, Rossi DJ. Reprogramming human fibroblasts to pluripotency using modified mRNA. Nat Protoc. 2013;8(3):568-582.

10. Yakubov E, Rechavi G, Rozenblatt S, Givol D. Reprogramming of human fibroblasts to pluripotent stem cells using mRNA of four transcription factors. Biochem Biophys Res Commun. 2010;394(1):189-193.

11. Kim D, Kim CH, Moon JI, et al. Generation of human induced pluripotent stem cells by direct delivery of reprogramming proteins. Cell Stem Cell. 2009;4(6):472-476.

12. Kormann MS, Hasenpusch G, Aneja MK, et al. Expression of therapeutic proteins after delivery of chemically modified mRNA in mice. Nat Biotechnol. 2011;29(2):154-157.

13. Bettinger T, Carlisle RC, Read ML, Ogris M, Seymour LW. Peptidemediated RNA delivery: a novel approach for enhanced transfection of primary and post-mitotic cells. Nucleic Acids Res. 2001; 29(18):3882-3891.

14. Wada R, Muraoka N, Inagawa K, et al. Induction of human cardiomyocyte-like cells from fibroblasts by defined factors. Proc Natl Acad Sci U S A. 2013;110(31):12667-12672.

15. Laeremans H, Rensen SS, Ottenheijm HC, Smits JF, Blankesteijn WM. Wnt/frizzled signalling modulates the migration and differentiation of immortalized cardiac fibroblasts. Cardiovasc Res. 2010;87(3): 514-523.

16. Subramanian M, Lim J, Dobson J. Enhanced nanomagnetic gene transfection of human prenatal cardiac progenitor cells and adult cardiomyocytes. PLoS One. 2013;8(7):e69812.
17. Drews K, Tavernier G, Demeester J, et al. The cytotoxic and immunogenic hurdles associated with non-viral mRNA-mediated reprogramming of human fibroblasts. Biomaterials. 2012;33(16):4059-4068.

18. Zhang L, Hoffman JA, Ruoslahti E. Molecular profiling of heart endothelial cells. Circulation. 2005;112(11):1601-1611.

19. Zhang H, Li N, Sirish P, et al. The cargo of CRPPR-conjugated liposomes crosses the intact murine cardiac endothelium. J Control Release. 2012;163(1):10-17.

20. Qian L, Berry EC, Fu JD, Ieda M, Srivastava D. Reprogramming of mouse fibroblasts into cardiomyocyte-like cells in vitro. Nat Protoc. 2013; 8(6):1204-1215.

21. Carey BW, Markoulaki S, Hanna JH, et al. Reprogramming factor stoichiometry influences the epigenetic state and biological properties of induced pluripotent stem cells. Cell Stem Cell. 2011;9(6):588-598.

22. Papapetrou EP, Tomishima MJ, Chambers SM, et al. Stoichiometric and temporal requirements of Oct4, Sox2, Klf4, and c-Myc expression for efficient human iPSC induction and differentiation. Proc Natl Acad Sci U S A. 2009;106(31):12759-12764.

23. Bisping E, Ikeda S, Sedej M, et al. Transcription factor GATA4 is activated but not required for insulin-like growth factor 1 (IGF1)-induced cardiac hypertrophy. J Biol Chem. 2012;287(13):9827-9834.

24. Bruneau BG, Nemer G, Schmitt JP, et al. A murine model of HoltOram syndrome defines roles of the T-box transcription factor Tbx 5 in cardiogenesis and disease. Cell. 2001;106(6):709-721. 


\section{Supplementary materials}

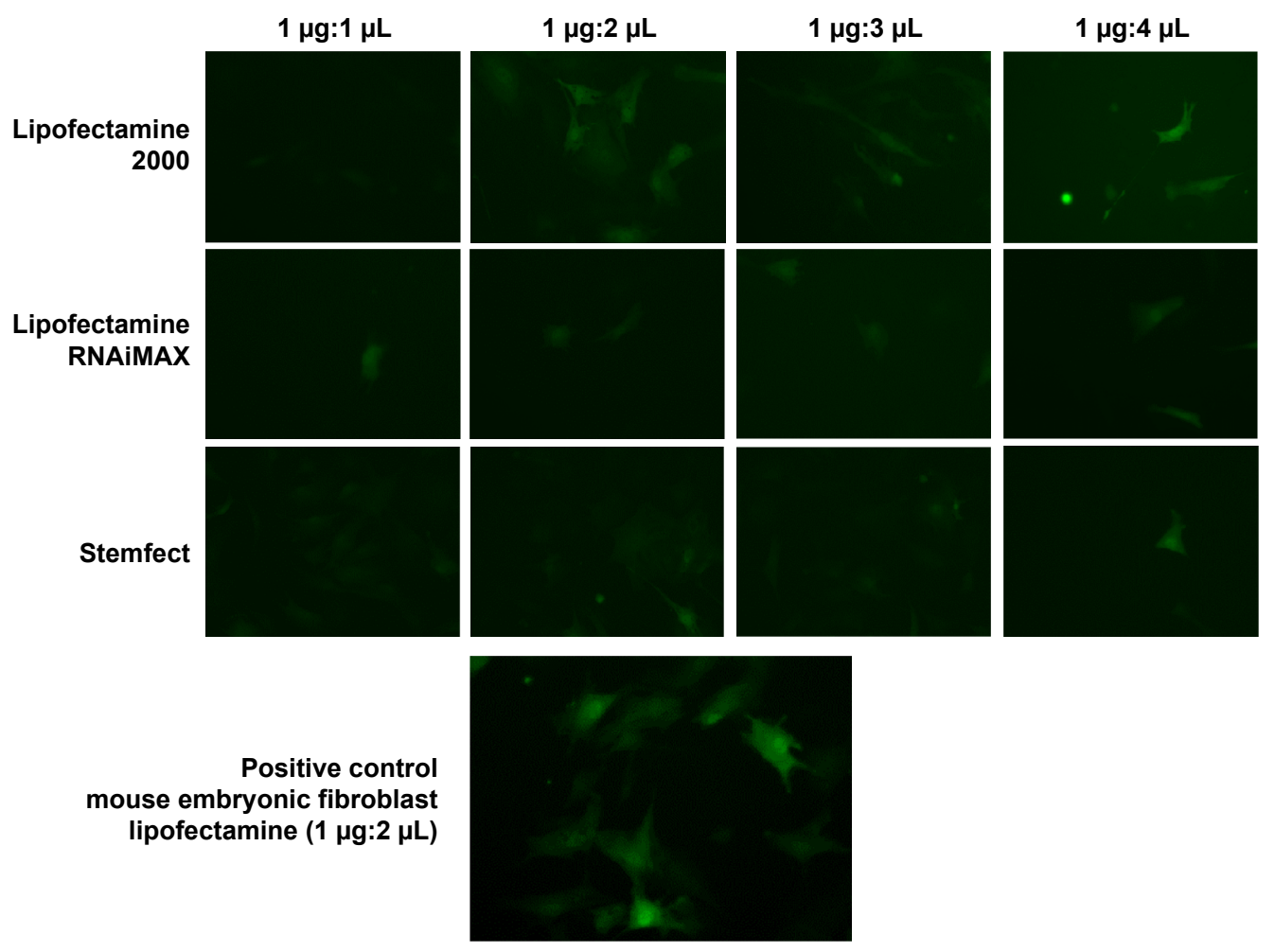

Figure SI eGFP mRNA transfection was optimized with various transfection reagents.

Notes: Various ratios of eGFP mRNA and transfection reagents were tested for cardiac fibroblast transfection. Compared to mouse embryonic fibroblasts that are easy to transfect with lipofectamine, cardiac fibroblasts show inefficient transfection in tested conditions. Among the tested transfection reagents, lipofectamine (I $\mu g \mathrm{mRNA}$ to $2 \mu \mathrm{L}$ lipofectamine) shows the best transfection efficiency. Fluorescence images were taken from a confluent 24 -well plate. Scale of width of each image is $50 \mu \mathrm{m}$. Fluorescence images of transfection reagents that had very inefficient transfection including TransIT-X2, TransIT-mRNA (Mirus Bio LLC, Madison, WI, USA), bPEI, and PEG-polylysine (22 ethylene glycol units, 50 lysine units) are not shown. Transfection was conducted as described in the main text.

Abbreviations: bPEl, branched polyethyleneimine; eGFP, enhanced green fluorescence protein.

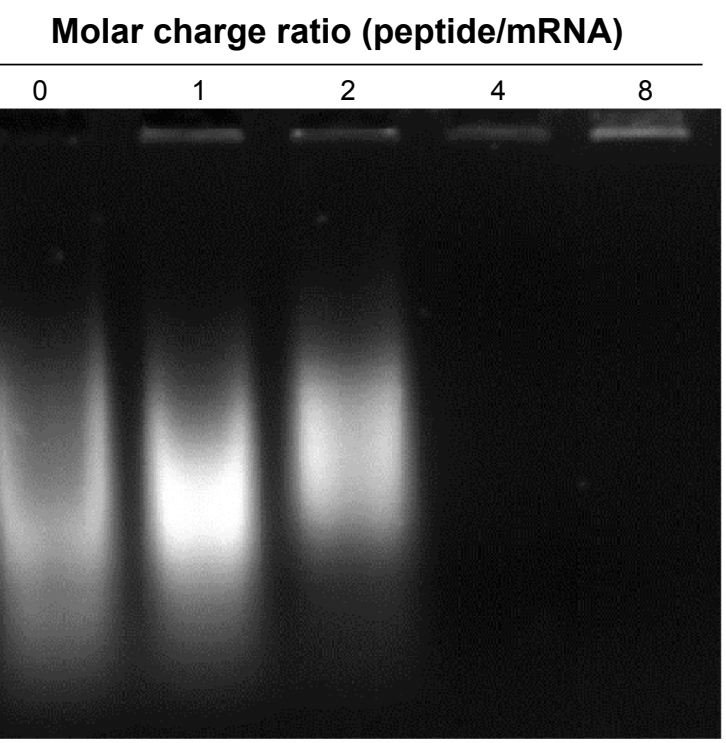

Figure S2 Electrophoretic mobility shift assay shows complexation of mRNA and CRPPR-R9.

Notes: EMSA was conducted to investigate if CRPPR-R9 complexes with mRNA. A mixture of CRPPR-R9 and mRNA produced visible retarded RNA bands from a molar charge ratio of one ([positive amines in peptide] to [phosphates in mRNA]) and a complete complexation was observed in a molar charge ratio of 4 or above. Unbound mRNA bands look smeared because different sizes of polyA tail ( $10-1,000 \mathrm{bp}$ ) were added to eGFP mRNA during polyA tailing.

Abbreviations: bp, base pairs; eGFP, enhanced green fluorescence protein; EMSA, electrophoretic mobility shift assay. 


\section{Positive control}

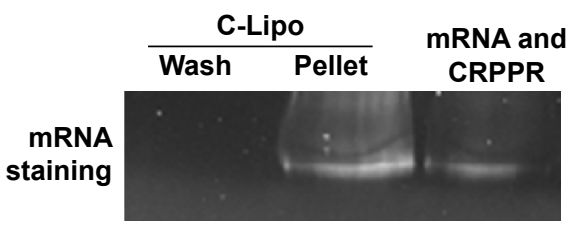

Peptide staining

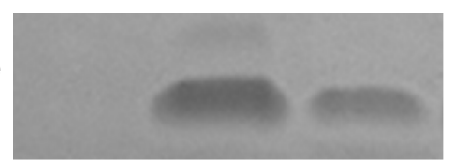

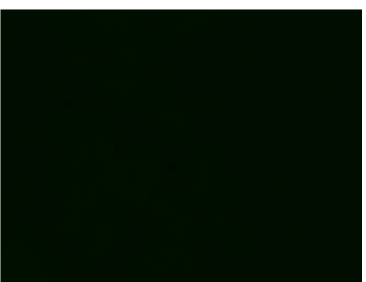

C-Lipo supernatant

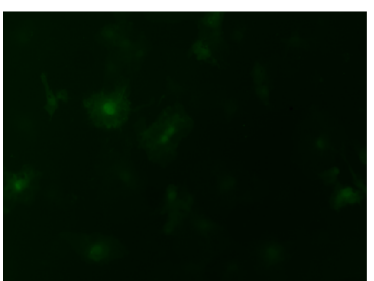

C-Lipo pellet
Figure S3 Gel electrophoresis after third wash step shows CRPPR-R9 incorporation in C-Lipo.

Notes: To study whether the CRPPR-R9 peptide forms a complex together with mRNA and lipofectamine, C-Lipo formation and gel electrophoresis were conducted. C-Lipo was formulated as described in the manuscript, centrifuged at $14,000 \times \mathrm{g}$ for 30 minutes, and the supernatant containing unbound CRPPR-R9 was removed. PBS ( $\mathrm{I} \mathrm{mL}$ ) was added to the solution, centrifugation repeated, and the supernatant removed. This wash step was conducted twice. During the third wash step, the pellet was redispersed with $20 \mu \mathrm{L}$ PBS, centrifuged, and the solution was carefully collected into pellet and supernatant for gel electrophoresis. The supernatant of the wash, liposome pellet, and positive control were analyzed with SDS-PAGE gel electrophoresis. The gel was stained with SYBR Green and Coomassie Blue. The third wash solution did not stain any mRNA or CRPPR, showing that the wash steps were properly conducted. Positive control of the mRNA and CRPPR peptide shows that C-Lipo contains mRNA and CRPPR peptide. Moreover, unlike the supernatan of the centrifuged C-Lipo that could not transfect cardiac fibroblasts, pelleted C-Lipo transfected cardiac fibroblasts and generated eGFP.The results prove that the CRPPR peptide is associated in C-Lipo. Scale of width of each image is $50 \mu \mathrm{m}$.

Abbreviations: eGFP, enhanced green fluorescence protein; PBS, phosphate buf fered saline; SDS-PAGE, sodium dodecyl sulfate polyacrylamide gel electrophoresis.

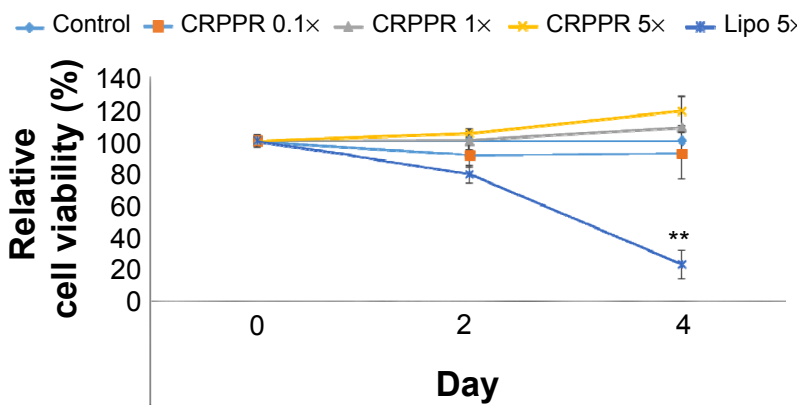

Figure S5 CRPPR-R9 does not affect cardiac fibroblast viability.

Notes: The cell viability of cardiac fibroblasts at various concentrations of CRPPRR9 was measured with CCK-8. CRPPR-R9 and lipofectamine concentrations were determined based on transfection conditions (CRPPR IX $=3.8 \mu \mathrm{g}$ of CRPPR-R9 per well of a 24 -well plate; Lipo $5 \times=5 \mu \mathrm{L}$ per well of a 24 -well plate). The result shows that CRPPR-R9 does not compromise cell viability even at five-fold higher concentration than the concentration used for transfection, whereas a similar concentration level of lipofectamine caused significant cell death in 4 days. Abbreviation: CCK-8, Cell Counting Kit-8.

\section{HeLa cell transfection}

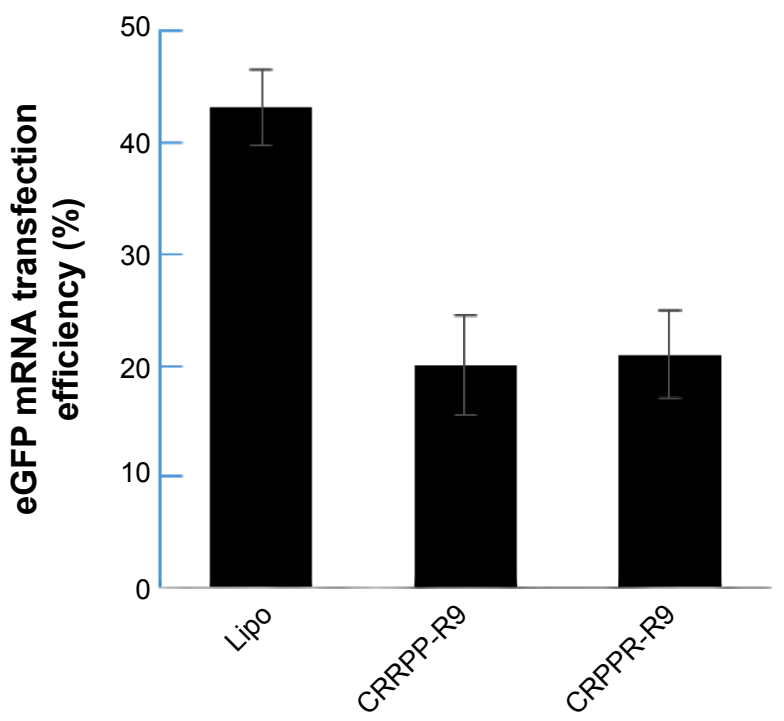

Figure S4 eGFP transfection efficiency shows that the CRPPR-R9 peptide does not improve lipofectamine transfection in HeLa cells, cervical carcinoma cells, which are not from cardiac origin.

Notes: eGFP mRNA transfection in HeLa cells was analyzed by flow cytometry. eGFP mRNA transfection efficiency of lipofectamine, CRPPR-R9/Lipo, and CRRPPR9(scramble)/Lipo are shown. Both CRRPP-R9 and CRPPR-R9 transfection with lipofectamine show even lower transfection efficiencies compared to the lipofectamine only transfection. The results suggest that the CRPPR-R9 peptide is not effective in HeLa cells that are not targeted by the CRPPR peptide sequence. We speculate that the very low transfection efficiency of peptide/lipofectamine in HeLa cells is due to a charge imbalance that interferes with the complexation of lipofectamine and mRNAs. The results represent mean $\pm S E, n=3$. CRPPR-R9 or CRRPP-R9 (3.8 $\mu \mathrm{g}, 20$ molar charge ratio) was added to the eGFP mRNA $(0.5 \mu \mathrm{g})$, and incubated at room temperature for 15 minutes. Next, I $\mu \mathrm{L}$ of lipofectamine 2000 in $50 \mu \mathrm{L}$ OptiMEM was added to the solution and further incubated for 15 minutes. Cardiac fibroblasts were transfected overnight as described earlier. At 24 hours post-transfection, flow cytometry analysis was conducted. Transfection efficiency was quantified based on the percentage of eGFP+ cardiac fibroblasts using InCyte software (EMD Millipore, Billerica, MA, USA).

Abbreviations: eGFP, enhanced green fluorescence protein; SE, standard error.

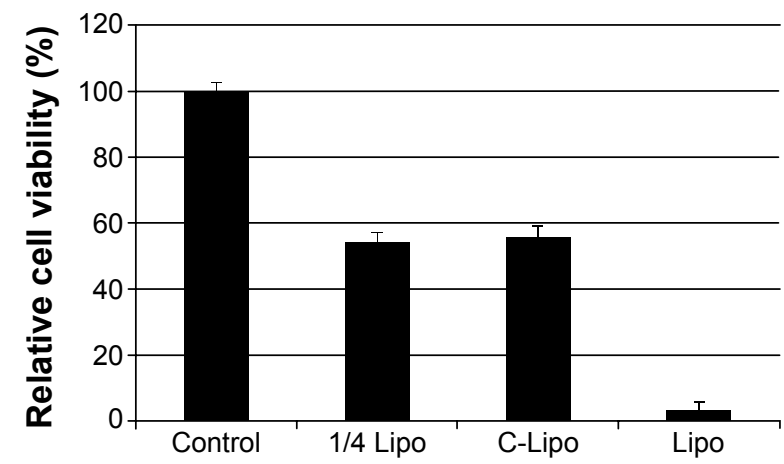

Figure S6 Low cytotoxicity of C-Lipo transfection is due to the reduced amount of lipofectamine.

Notes: Cell viability of transfected cardiac fibroblasts was measured on day 14 from the initial single transfection. Transfection was conducted with GMT mRNAs as described in the main manuscript. One-quarter of the Lipo sample contained one-quarter of the lipofectamine amount used for Lipo. The C-Lipo and I/4 Lipo transfections showed similar levels of cell viability. This result suggests that the high cell viability of the C-Lipo transfection is mainly due to the reduced amount of lipofectamine. Abbreviation: GMT, Gata4, Mef2c, and Tbx5. 
Table SI Primer list

\begin{tabular}{lll}
\hline Actcl & Forward & TTTGCGGATATCGATGTCACACTTC \\
Actn2 & Reverse & ACTGTGTTACGTCGCCCTGGAT \\
& Forward & AGGGGATTGTGCGCCGAATC \\
Gjal & Reverse & AGCTAACAGAATATGTAAGGT \\
& Forward & AGCAGCAGACTTTGAAACTTTAAA \\
Hand2 & Reverse & CCGACAGCCACACCTTCCCT \\
& Forward & GACGTGAAAGAGGAGAAGA \\
Nppa & Reverse & GTGCTGTGGCCAGCCTGTCCGG \\
& Forward & AACCAGAGTGGGCAGAGACAG \\
Tnnt2 & Reverse & TGTGTTGGACACCGCACTGTA \\
& Forward & GTGTGCAGTCCCTGTTCAGA \\
\hline
\end{tabular}

\section{Publish your work in this journal}

The International Journal of Nanomedicine is an international, peerreviewed journal focusing on the application of nanotechnology in diagnostics, therapeutics, and drug delivery systems throughout the biomedical field. This journal is indexed on PubMed Central, MedLine, CAS, SciSearch ${ }^{\circledR}$, Current Contents ${ }^{\circledR} /$ Clinical Medicine,
Journal Citation Reports/Science Edition, EMBase, Scopus and the Elsevier Bibliographic databases. The manuscript management system is completely online and includes a very quick and fair peer-review system, which is all easy to use. Visit http://www.dovepress.com/ testimonials.php to read real quotes from published authors. 